\title{
VEGF Trap In Combination With Radiotherapy Improves Tumor Control In U87 Glioblastoma
}

\author{
Phyllis Wachsberger, $\mathrm{PhD}$ \\ Thomas Jefferson University, phyllis.wachsberger@jefferson.edu \\ Randy Burd, PhD \\ Department of Nutritional Sciences, University of Arizona, Tuscon AZ
}

Chris Cardi, MS

Department of Radiology, Thomas Jefferson University, chris.cardi@jefferson.edu

Mathew L. Thakur

Thomas Jefferson University, mathew.thakur@jefferson.edu

Constantine Daskalakis

Eollow this and additional works at: https://jdc jefferson.edu/bodinejournal

Thomas JeffersonUniversity, Constantine.Daskalakis@jefferson.edu

Part of the Oncology Commons

Let us know how access to this document benefits you
see next page for adaitional authors

\section{Recommended Citation}

Wachsberger, PhD, Phyllis; Burd, PhD, Randy; Cardi, MS, Chris; Thakur, Mathew L.; Daskalakis, Constantine; Holash, PhD, Jocelyn; Yancopoulos, PhD, George D.; and Dicker, Adam MD, PhD (2008) "VEGF Trap In Combination With Radiotherapy Improves Tumor Control In U87 Glioblastoma," Bodine Journal: Vol. 1 :

Iss. 1 , Article 4.

DOI: https://doi.org/10.29046/TBJ.001.1.003

Available at: https://jdc.jefferson.edu/bodinejournal/vol1/iss1/4

This Article is brought to you for free and open access by the Jefferson Digital Commons. The Jefferson Digital Commons is a service of Thomas Jefferson University's Center for Teaching and Learning (CTL). The Commons is a showcase for Jefferson books and journals, peer-reviewed scholarly publications, unique historical collections from the University archives, and teaching tools. The Jefferson Digital Commons allows researchers and interested readers anywhere in the world to learn about and keep up to date with Jefferson scholarship. This article has been accepted for inclusion in Bodine Journal by an authorized administrator of the Jefferson Digital Commons. For more information, please contact: JeffersonDigitalCommons@jefferson.edu. 


\section{VEGF Trap In Combination With Radiotherapy Improves Tumor Control In U87 Glioblastoma}

\section{Authors}

Phyllis Wachsberger, PhD; Randy Burd, PhD; Chris Cardi, MS; Mathew L. Thakur; Constantine Daskalakis; Jocelyn Holash, PhD; George D. Yancopoulos, PhD; and Adam Dicker MD, PhD 


\section{VEGF Trap In Combination With Radiotherapy Improves Tumor Control In U87 Glioblastoma}

\author{
Phyllis R. Wachsberger, PhD, ${ }^{\star}$ Randy Burd, PhD, ${ }^{\S}$ Chris Cardi, MS, ${ }^{\dagger}$ \\ Mathew Thakur, PhD, ${ }^{\dagger}$ Constantine Daskalakis, ScD, ${ }^{\ddagger}$ Jocelyn Holash, PhD, \\ George D. Yancopoulos, PhD," And Adam P. Dicker, MD, PhD, ${ }^{*}$ \\ Departments of *Radiation Oncology, ${ }^{\dagger}$ Radiology, †Pharmacology and Experimental Therapeutics, \\ Thomas Jefferson University, Philadelphia, PA; ${ }^{\S}$ Department of Nutritional Sciences, University of \\ Arizona, Tucson, AZ; and "Novartis, Emeryville, CA
}

The following article is reprinted with permission from Elsevier Inc. It was originally published in the Int. J. Radiation Oncol. Biol. Physics, Volume 67, Issue 5, pages 1526-1537, 2007.

\section{Purpose}

To determine the effect of vascular endothelial growth factor VEGF Trap (Regeneron Pharmaceuticals, Tarrytown, NY), a humanized soluble vascular endothelial growth factor (VEGF) receptor protein, and radiation (RT) on tumor growth in U87 glioblastoma xenografts in nude mice.

\section{Methods and Materials}

U87 cell suspensions were implanted subcutaneously into hind limbs of nude mice. VEGF Trap $(2.5-25 \mathrm{mg} / \mathrm{kg})$ was administered every 3 days for 3 weeks alone or in combination with a single dose of 10 Gy or fractionated RT ( 3 x 5 Gy). In addition, three scheduling protocols for VEGF Trap plus fractionated RT were examined.

\section{Results}

Improved tumor control was seen when RT (either single dose or fractionated doses) was combined with the lowest dose of VEGF Trap $(2.5 \mathrm{mg} / \mathrm{kg})$. Scheduling did not significantly affect the efficacy of combined therapy. Although high-dose VEGF Trap (10 mg/kg or $25 \mathrm{mg} / \mathrm{kg}$ ) significantly reduced tumor growth over that of RT alone, there was no additional benefit to combining high-dose VEGF Trap with RT.

\section{Conclusions}

Vascular endothelial growth factor Trap plus radiation is clearly better than radiation alone in a U87 subcutaneous xenograft model. Although high doses of VEGF Trap alone are highly efficacious, it is unclear whether such high doses can be used clinically without incurring normal tissue toxicities. Thus, information on lower doses of VEGF Trap and ionizing radiation is of clinical relevance. () 2007 Elsevier Inc.

Key Words: Vascular endothelial growth factor Trap, Radiotherapy, Anti-angiogenic, U87 glioblastoma.

\section{Introduction}

Radiation (RT) therapy is an important treatment modality for many cancers; however, its therapeutic success is impeded by dose-limiting normal tissue toxicities and the development of radioresistance. Recent studies emphasize the importance of the tumor microvascular response in addition to the tumor cell response in determining tumor radioresistance ${ }^{1,2}$. Ionizing radiation can directly induce endothelial cell apoptosis ${ }^{1,3}$, which can inhibit tumor growth and lead to radiosensitization. However, in opposition to endothelial cell damage, radiation also induces signal transduction cascades, which contribute to radiation resistance through upregulation of proliferative, survival, and angiogenic pathways ${ }^{4}$. In particular, radiation induces vascular cytokines, such as vascular endothelial growth factor $(\mathrm{VEGF})^{5,6}$, one of the most potent endothelial cell survival factors ${ }^{7}$, which functions as a powerful antiapoptotic factor for endothelial cells in new blood vessels ${ }^{8,9}$. Radiation-induced VEGF results in tumor radioresistance through vascular radioprotection ${ }^{2,10}$.
Inhibition of VEGF activity or disabling the function of VEGF receptors is therefore a potential strategy for improving radiation outcome. The VEGF blockade alone has been shown to inhibit both tumor growth and metastasis in a variety of animal tumor models ${ }^{11}$. Currently, three approaches are in clinical development to target the VEGF/VEGFR-signaling pathway: (1) monoclonal antibodies directed against VEGF or its receptors ${ }^{12-15}$, (2) small molecule inhibitors of the VEGFR2 tyrosine kinase enzyme ${ }^{16-19}$, and (3) soluble decoy receptors created from the VEGFR1 receptor which selectively inhibit VEGF $^{20,21}$. The relative benefits of these strategies have yet to be determined clinically.

Tumor cures are rare when VEGF blockers are used as the sole method of treatment; in general, antiangiogenics appear to work best in combination with cytotoxic therapies ${ }^{22}$. A number of preclinical studies suggest that radiotherapy in combination with VEGF targeting agents enhances the radiotherapeutic ratio (see reviews; ${ }^{23,24}$ ). The best way to incorporate VEGF inhibition strategies into current radiotherapy regimens remains unknown.

Because of the role that angiogenesis plays in the radiation response, the objective of this study was to determine whether VEGF Trap (Regeneron Pharmaceuticals, Tarrytown, NY), a potent anti-VEGF angiogenesis inhibitor that traps circulating VEGF in the bloodstream and in the extracellular space, would enhance radiation therapy in the human U87 glioblastoma (GBM) tumor model. Because GBM tumors are among the most radioresistant and vascular of neoplasms and are known to secrete high levels of VEGF ${ }^{25}$, U87 GBM was deemed an appropriate model to assess the effects of VEGF Trap and radiation. It was hypothesized that inhibition of VEGF signaling by VEGF Trap would improve the human U87 glioblastoma model response to radiotherapy.

The administration of decoy soluble VEGF receptors has been found to be a very effective way to block the VEGF signaling pathway ${ }^{26-29}$. VEGF Trap is a unique human fusion protein comprising portions of human VEGF recep- 
tor 1 (VEGFR1) and human VEGF receptor 2 (VEGFR2) extracellular domains fused to the constant region $(\mathrm{Fc})$ of human IgG1 ${ }^{21}$. VEGF Trap has greater affinity for the VEGF ligand than anti-VEGF monoclonal antibodies (mab) do (dissociation constant $<1 \mathrm{pMol} / \mathrm{L}$ for VEGF Trap vs. $0.1-10 \mathrm{nMol} / \mathrm{L}$ for mab) ${ }^{30}$. VEGF Trap has been shown to inhibit neoangiogenesis and tumor growth in tumor xenografts and metastases, as well as reduce the formation of malignant ascites ${ }^{14,21,31}$.

\section{Methods and Materials}

Analysis of VEGF levels in U87 tumor cells in culture

U87 glioblastoma cells (American Type Culture Collection) were maintained in alpha MEM (Sigma-Aldrich, St. Louis, MO) with 10\% fetal bovine serum (Atlanta Biologicals, Norcross, GA). U87 cells were irradiated at doses between 2 and $20 \mathrm{~Gy}$ in the presence or absence of $40 \mathrm{nM}$ VEGF Trap and incubated for $48 \mathrm{~h}$. Using a commercially available human VEGF immunoassay kit (R\&D Systems, Minneapolis, MN), VEGF was assayed from culture supernatants.

\section{Animal and tumor model}

U87 cell suspensions ( $5 \times 10^{5}$ cells in $100 \mu \mathrm{L}$ phosphate buffered saline) were implanted subcutaneouly (SC) into the right hind limbs of athymic NCR NUM mice (Taconic Farms, Hudson, NY). A SC xenograft model was chosen to facilitate radiation dosing and ease of tumor measurements in the more than 200 mice measured in this study. Mice were not pretreated before tumor implantation. U87 tumors were allowed to grow for approximately 14 to 18 days until reaching an approximate diameter of 4 to $5 \mathrm{~mm}$ before treatment.

\section{Drug and irradiation treatment}

In an initial pilot study, VEGF Trap was administered at two doses, a high dose $(25 \mathrm{mg} / \mathrm{kg})$ or low dose $(2.5 \mathrm{mg} / \mathrm{kg})$, every 3 days, up to 3 weeks, with or without a single dose of radiation (10 Gy) given on Day 0. VEGF Trap was administered every 3 days because it has a half-life of $72 \mathrm{~h}$ in mouse serum (drug pharmacokinetics communicated by Regeneron). Drug was administered $2 \mathrm{~h}$ before radiation. When fractionated radiotherapy was used, VEGF Trap was combined at $2.5 \mathrm{mg} / \mathrm{kg}$ (low dose) or $10 \mathrm{mg} / \mathrm{kg}$ (intermediate dose) with fractionated radiotherapy (three fractions of 5 Gy each) on Days 0, 1, and 2. Scheduling of VEGF Trap was either 1 week before fractionated radiation and continuing for a period of 3 weeks, concurrent with radiation and continuing for a period of 3 weeks, or 3 days postradiation treatment and continuing for a period of 3 weeks. Thus, the total number of drug doses was constant for each schedule (see Fig. 1 for dose and irradiation scheduling protocol).

Irradiation was performed on anesthetized mice using an X-ray machine (Gulmay Medical, Bethel, CT) operating at $250 \mathrm{kV}, 10 \mathrm{~mA}$, with a $2-\mathrm{mm}$ aluminum filtration. The effective photon energy was $\approx 90 \mathrm{keV}$. Mice were anesthetized with a combination of ketamine and acepromazine at a concentration of $75 \mathrm{mg} / \mathrm{kg}$ and $0.35 \mathrm{mg} / \mathrm{kg}$, respectively. Each mouse was confined in a lead casing with its tumor-bearing leg extended through an opening on the side to allow the tumor to be irradiated locally. Radiation was administered as three daily fractions of 5 Gy each as described earlier.

Tumor size was measured 4 to 5 times per week after treatment by direct measurement with calipers and calculated by the formula [(smallest diameter (2) $\mathrm{x}$ widest diameter) / 2]. Tumors were not allowed to grow beyond 2,000 $\mathrm{mm}^{3}$ in accordance with Institutional Animal Care and Use Committee regulations.

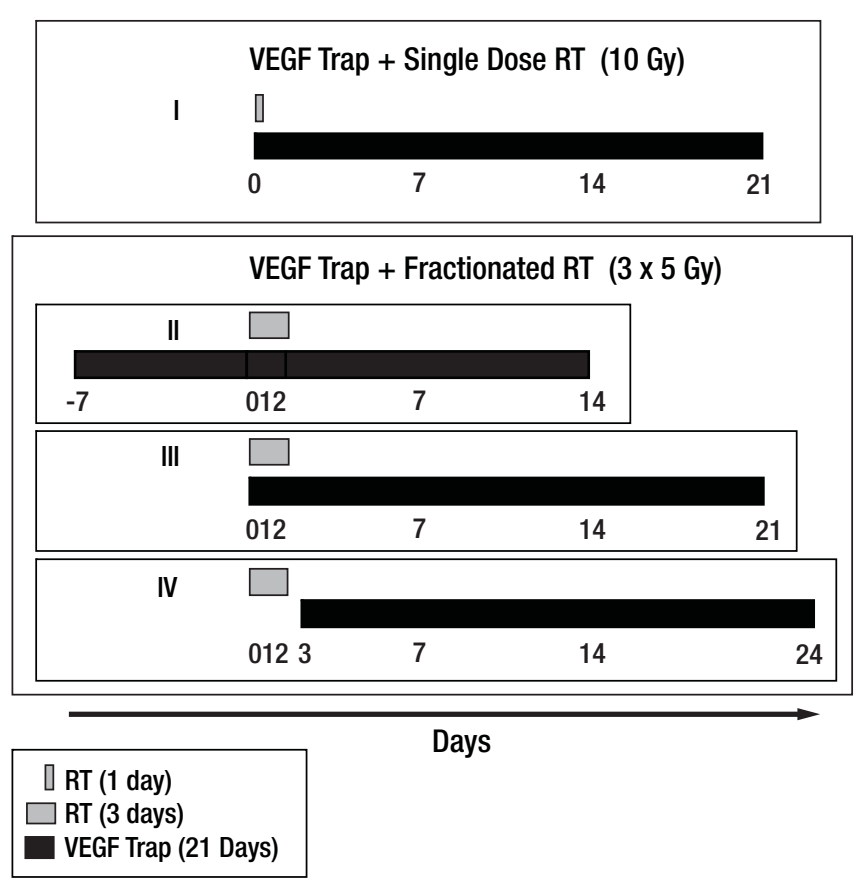

Figure 1. Scheduling protocols for vascular endothelial growth factor (VEGF) Trap administration in combination with radiation (RT). VEGF Trap was given at $2.5,10$, or $25 \mathrm{mg} / \mathrm{kg}$ every 3 days in four schedules: (I) VEGF Trap given on Day 0 concurrent with a single dose of RT (10 Gy) and continued up to 3 weeks; (II) VEGF Trap given on day -7 before RT ( 3 x 5 Gy) and continued for 3 weeks; (III) VEGF Trap given on Day 0 concurrent with RT ( 3 x 5 Gy) and continued up to 3 weeks; (IV) VEGF Trap given on Day 3 post RT ( 3 x 5 Gy) and continued up to 3 weeks. All three protocols received the same number of drug doses. Day 0 was always the start of radiation.

\section{Positron emission tomography imaging}

The MOSAIC PET scanner (Philips Medical Systems, Brisbane, CA) was used for PET studies. Before imaging, mice were anesthetized with ketamine $(75 \mathrm{mg} / \mathrm{kg})$ and acepromazine $(0.35 \mathrm{mg} / \mathrm{kg})$ via a SC injection. Once anesthetized 0.3 to $0.5 \mu \mathrm{Ci}$ of 18 -fluorodeoxyglucose (FDG) was administered intravenously. Sixty to seventy min were allowed for uptake of the tracer. Mice were placed in a 50-mL specimen tube to facilitate multimodality stereotactic positioning. The PET data were acquired in a single position for $15 \mathrm{~min}$. Volumes of interest (VOIs) were defined by drawing multislice regions of interest (ROIs) on the PET images using $50 \%$ of the full-width-at-half-maximum (FWHM) of the tumor to determine the tumor boundary. In the case of tumors with a core lacking FDG uptake, the tumor and core boundaries were defined by $50 \%$ FWHM of each wall adjacent to the core. Mice were divided into three groups ( $n$ = 3-6 animals per group): untreated; low-dose VEGF Trap-treated (2.5 $\mathrm{mg} / \mathrm{kg}$ ), and highdose VEGF Trap-treated (10 mg/kg). 


\section{Immunohistochemistry}

Platelet-endothelial cell adhesion molecule 1 (PECAM-1) immunostaining for microvessel density (MVD): control, radiation-treated, VEGF Trap-treated tumors, and VEGF Trap plus radiation-treated tumors were immunostained with a rat antimouse PECAM-1 mAb (BD Biosciences, Boston, MA) and a rabbit antirat biotinylated secondary antibody (Vector Labs, Burlingame, CA). Enhanced horseradish peroxidase-conjugated streptavidin and a substrate chromogen, AEC (3-amino-9-ethyl carbazole), were used to visualize the signal. (HISTOSTAIN-PLUS kit, Invitrogen, Carlsbad, CA); slides were examined with a Nikon Eclipse E600 microscope to calculate MVD, the area occupied by the PECAM-1-positive microvessels, and total tissue area per section were quantified using National Institute of Health Image J software. Microvessel density was expressed as percent area of blood vessels stained per tissue section. Areas of necrosis were excluded from calculations. Four or five high-power fields were identified on each section with three to four sections per tumor and two tumors per endpoint.

\section{Statistical analysis of tumor growth}

Tumor size measurements over time were obtained from the following groups: control; radiation alone; VEGF Trap, low dose ( $2.5 \mathrm{mg} / \mathrm{kg})$, intermediate dose $(10 \mathrm{mg} / \mathrm{kg})$, or high dose $(25 \mathrm{mg} / \mathrm{kg})$; and the corresponding two radiation plus VEGF Trap combinations $(n=10-14$ animals per group). Tumor growth over the entire study follow-up period was modeled via mixed-effects linear regression. This approach fits a "random" growth curve to each animal's data and then statistically "averages" these curves within each treatment group to estimate an overall "fixed effect" for each group. It also properly handles unbalanced data (i.e., different number of measurements for different animals) and takes into account the correlation of each animal's measurements over time. Because tumors typically grow exponentially, the base- 10 logarithm of tumor volume was modeled as a function of time and treatment. The interpretation of the linear model for the log of tumor volume is in terms of geometric means and geometric mean ratios (while the usual interpretation of a regression model for an untransformed outcome is in terms of arithmetic means and mean differences). The fitted linear growth curves fitted the data well. In addition, an allowance was made for the variance of the random effects to differ across groups to account for the larger variability of measurements in certain treated groups. All statistical analyses were conducted in SAS 8.2 (SAS Institute, Cary, NC, 1999-2001).

The mixed-effects regression has multiple advantages over analyses of tumor growth delay that typically compare groups with respect to the average time it takes tumors to reach some arbitrary size (e.g., 2,000 $\mathrm{mm} 3$ ). First, mixed-effects regression yields more general parameters of interest, such as average daily tumor growth rate and doubling time. Second, it can investigate (if necessary) treatment interactions and nonlinear patterns of tumor growth. Finally, it is more efficient because it used the repeated tumor size measurements obtained over the entire study period.

\section{Results}

Effect of VEGF Trap and radiation on VEGF secretion in U87 cells in culture

Levels of VEGF increased in U87 culture supernatants in a dose-dependent manner following irradiation (Fig. 2). The addition of VEGF Trap $(40 \mathrm{nM})$ reduced free VEGF in the supernatant to undetectable levels.

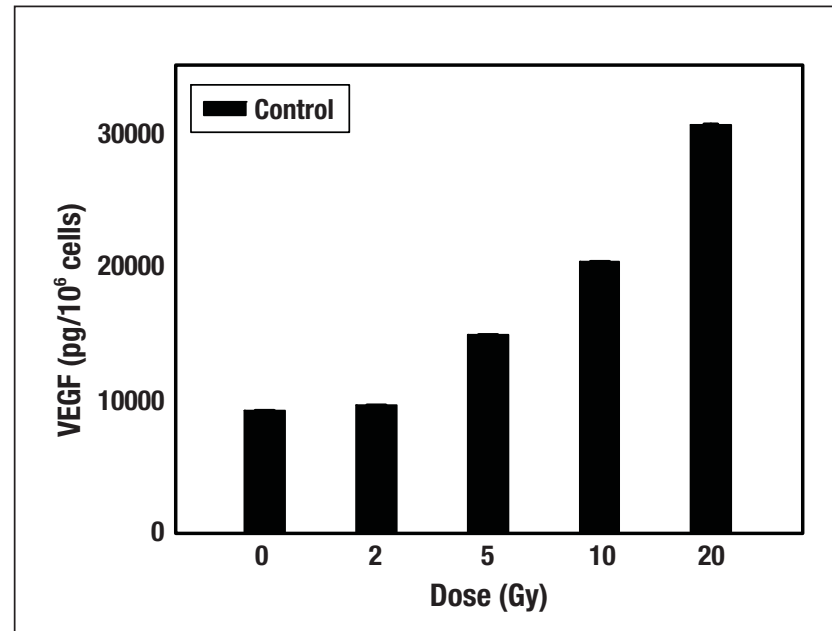

Figure 2. Effect of vascular endothelial growth factor (VEGF) Trap and radiation on VEGF secretion in U87 cells in culture. U87 cells were irradiated at doses between 2 and 20 Gy in the presence or absence of $40 \mathrm{nM}$ VEGF Trap. Cell culture supernatants were assayed for VEGF secretion $48 \mathrm{~h}$ following treatment. VEGF secretion was undetectable in presence of 40 nM VEGF Trap.

Effect of VEGF Trap and radiation on U87 tumor growth inhibition The linear models for the log-transformed tumor growth fitted the data quite well in all groups. The raw data for all treatment groups with regression lines are plotted in Figs. 3 through 6 with corresponding Tables 1 through 4 . The average daily percent increase in tumor volume for the untreated control group was consistent across all protocols and ranged between $27 \%$ and $31 \%$, corresponding to a tumor doubling time between 2.5 and 3.0 days (Tables 1-4). Radiation alone (both single or fractionated doses) or VEGF Trap alone (all doses) significantly reduced the tumor growth rate compared with control ( $p<0.001$, Figs. 3-6, Tables 1-4). Results with VEGF Trap in combination with single dose or fractionated radiotherapy are now summarized.

\section{Effect of VEGF Trap and single dose radiation (10 Gy) on U87 tumor growth inhibition}

Table 1 presents tumor growth data based on the mixed-effects linear regression analysis described in Methods and Materials, and Fig. 3 presents the original animal data. In this experiment, a low dose of VEGF $\operatorname{Trap}(2.5 \mathrm{mg} / \mathrm{kg})$ initiated concurrently with a single dose of $10 \mathrm{~Gy}$ was compared with a 10x higher dose of VEGF Trap (25 mg/kg) plus $10 \mathrm{~Gy}$. The six groups are compared in terms of average daily tumor growth and doubling time. It can be seen from Table 1 and Fig. 3 that both lowdose and high-dose VEGF Trap were effective inhibitors of daily percent increase in tumor volume $(\% \Delta=15 \%$ and $5 \%$, respectively, vs. $31 \%$ for controls, $p=0.001$ ). Although low-dose VEGF Trap was not significantly better than 10-Gy treatment alone, the combination of low-dose VEGF Trap and 10 Gy slowed daily tumor growth ( $\% \Delta=12 \%$ vs. $18 \%$ for 10 Gy alone and $15 \%$ for low VEGF Trap alone). Thus, a less than additive enhancement in tumor control over either modality alone was observed. High-dose VEGF Trap, as a single treatment modality, was highly effective in slowing daily percent increase in tumor volume (5\% vs. $18 \%$ for $10 \mathrm{~Gy})$. Its efficacy was not improved by the addition of $10 \mathrm{~Gy}$. This study 

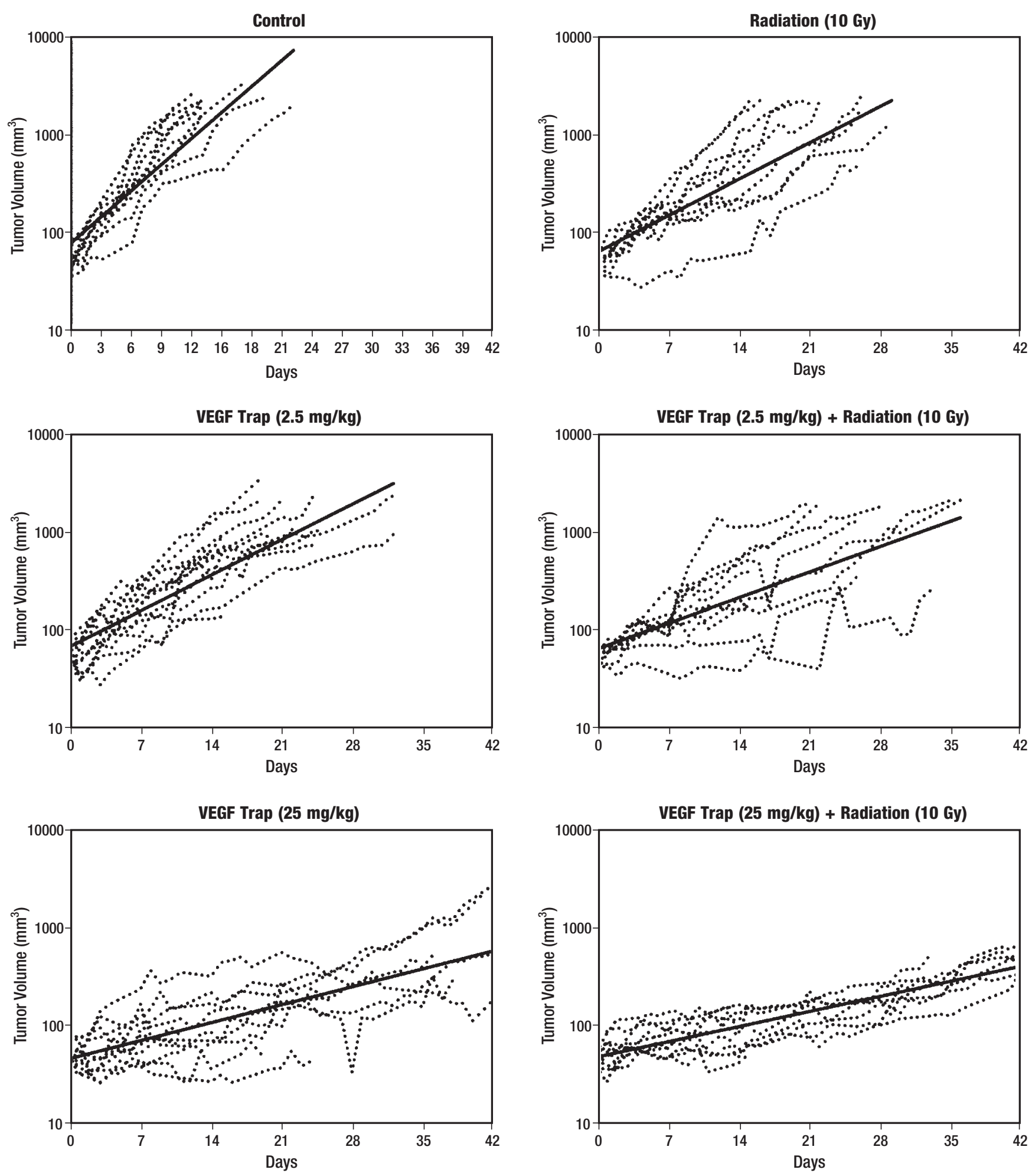

Figure 3. Effect of vascular endothelial growth factor (VEGF) Trap combined with single-dose radiation (10 Gy) on tumor growth in U87GBM. Individual mouse data for six treatment groups ( $n=10-12$ animals per group). VEGF Trap was given at 2.5 or $25 \mathrm{mg} / \mathrm{kg}$ starting on Day 0 , concurrent with radiation and continuing every 3 days for 3 weeks (see schedule I, Fig. 1). 

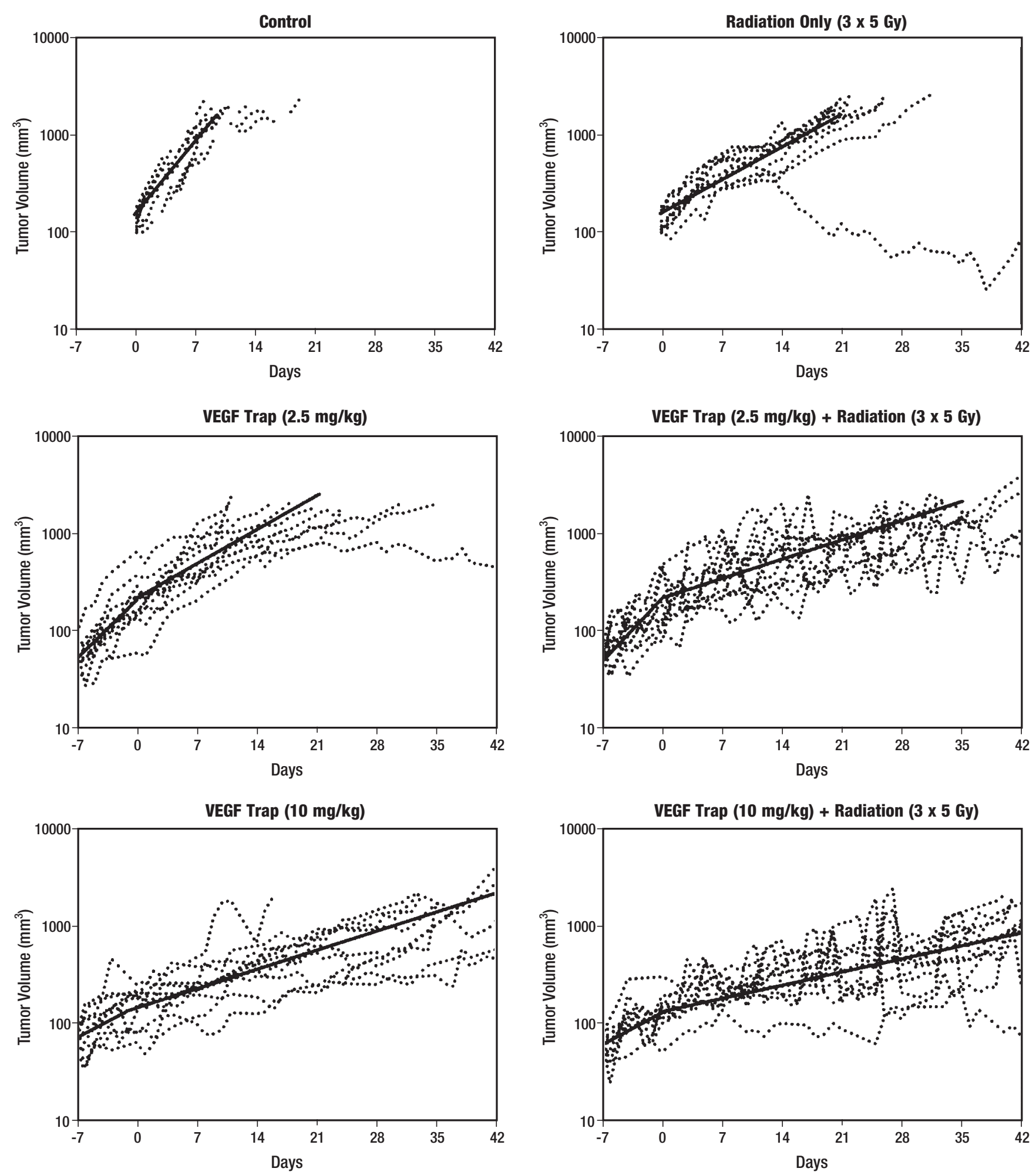

Figure 4. Effect of vascular endothelial growth factor (VEGF) Trap initiated before fractionated radiation ( $3 \times 5$ Gy) on tumor growth in U87 GBM. Individual mouse data for 6 treatment groups ( $n=10-14$ animals/group). VEGF Trap was given at 2.5 or $10 \mathrm{mg} / \mathrm{kg}$ starting on Day -7 and continuing every 3 days for 3 weeks (see schedule II, Fig. 2). 

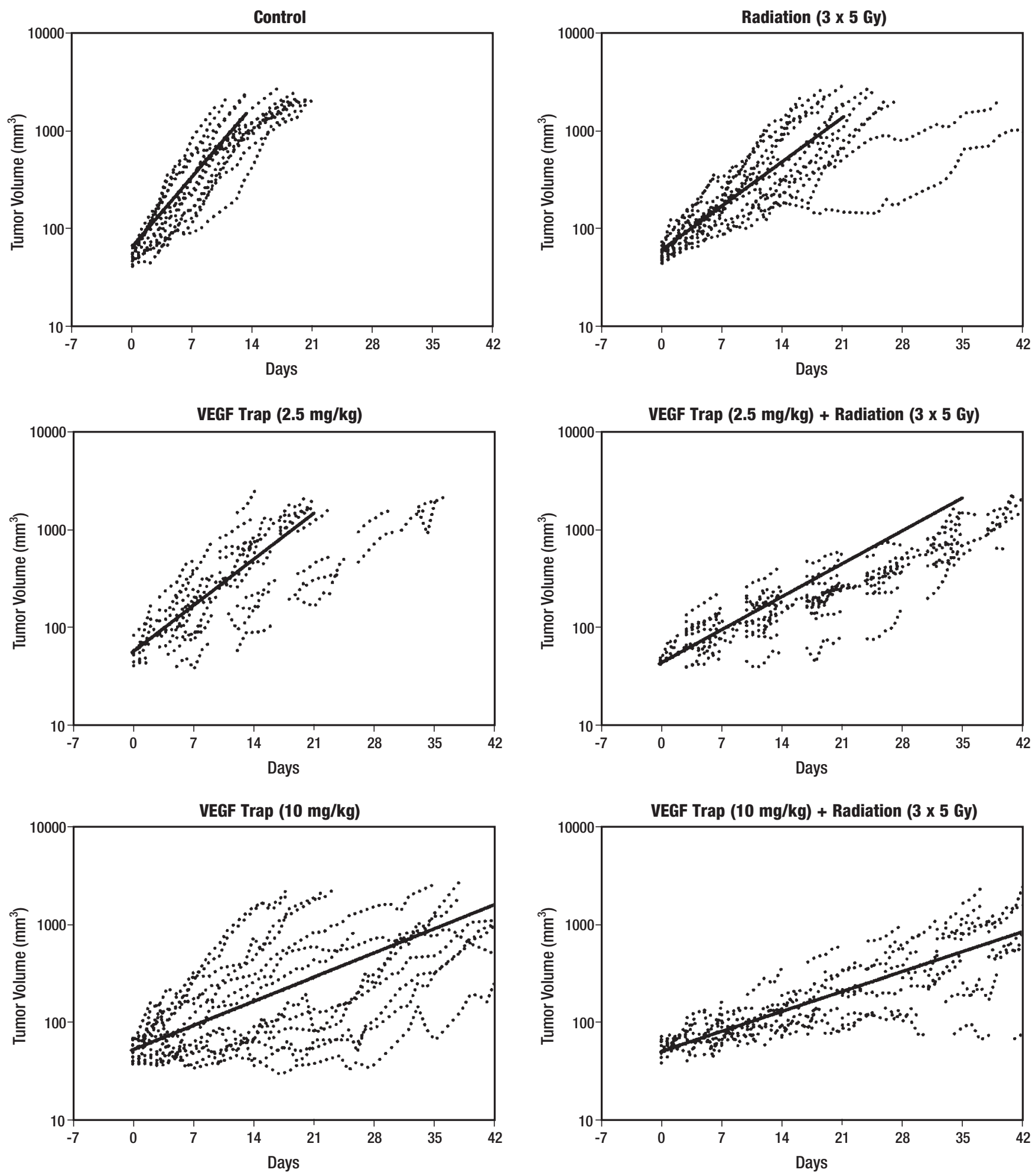

Figure 5. Effect of vascular endothelial growth factor (VEGF) Trap sequenced concurrent with fractionated radiation ( 3 x 5 Gy) on tumor Growth in U87 GBM. Individual mouse data for six treatment groups ( $\mathrm{n}=10-14$ animals per group). VEGF Trap was given at $2.5 \mathrm{or} 10 \mathrm{mg} / \mathrm{kg}$ starting on Day 0 and continuing every 3 days for 3 weeks (see schedule III, Fig. 3). 

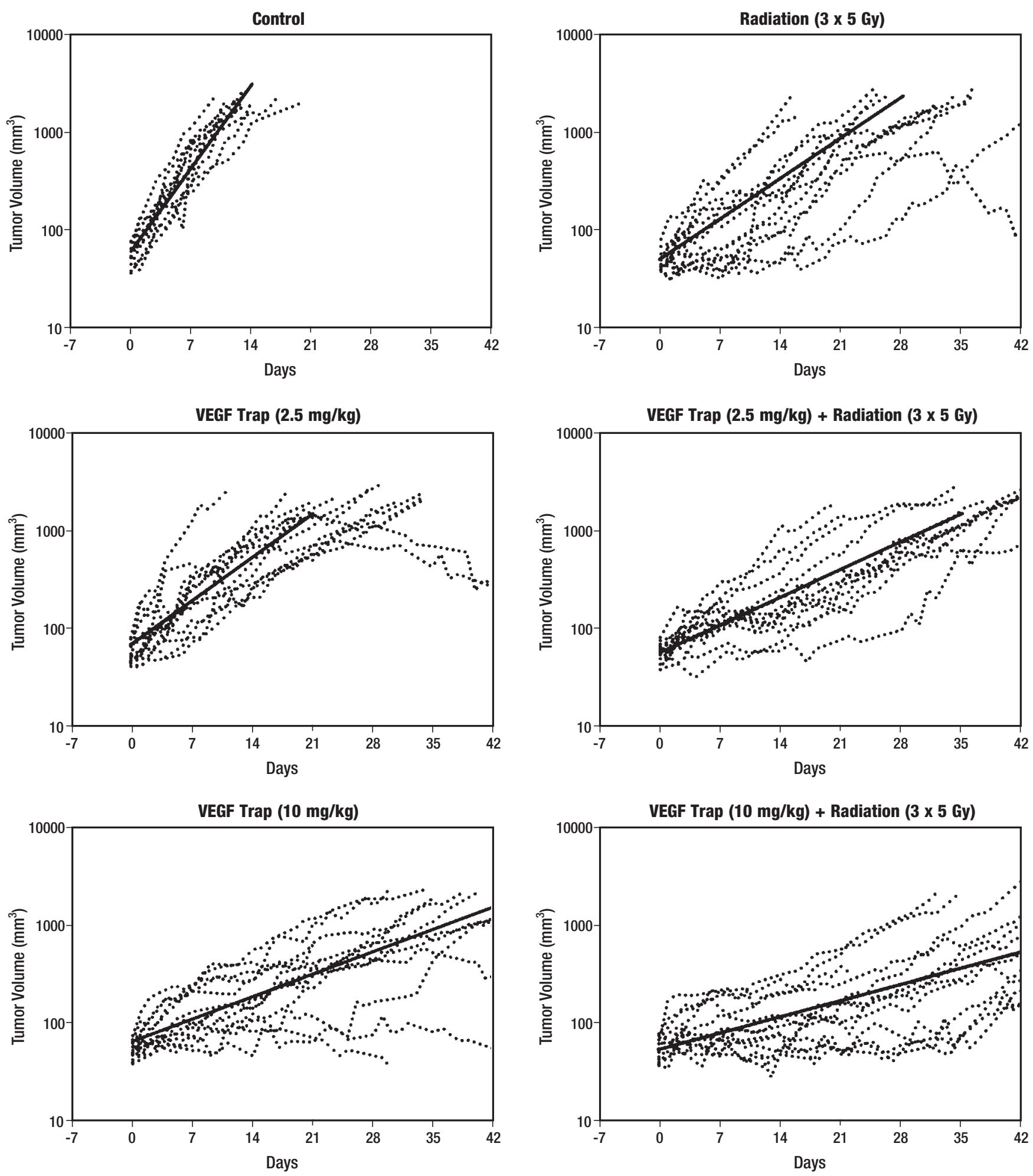

Figure 6. Effect of vascular endothelial growth factor (VEGF) Trap sequenced post-fractionated radiation ( 3 x 5 Gy) on tumor Growth in U87 GBM. Individual mouse data for six treatment groups ( $n=10-14$ animals/group). VEGF Trap was given at 2.5 or $10 \mathrm{mg} / \mathrm{kg}$ starting on Day 3 and continuing every 3 days for 3 weeks (see schedule IV, Fig. 4). 
Table 1. Effect of VEGF Trap combined with single-dose radiation: Summary of tumor growth (Schedule I)

\begin{tabular}{lclll}
\hline Treatment & $\% \Delta$ & $\mathbf{( 9 5 \%} \mathbf{C I})$ & T2x & \multicolumn{1}{c}{$\boldsymbol{p}$ values } \\
\hline Control (human FC protein) & 31.0 & $(27-35)$ & 2.6 & \\
\hline RT (10 Gy) & 18.0 & $(15-21)$ & 4.2 & 0.001 vs. control, 0.19 vs. VEGF Trap (low) \\
\hline VEGF Trap (2.5 mg/kg) & 15.0 & $(13-28)$ & 4.9 & 0.001 vs. control, 0.19 vs. RT alone \\
\hline VEGF Trap (25 mg/kg) & 5.0 & $(2-7)$ & 15.2 & 0.001 vs. control, 0.001 vs. RT alone, 0.001 vs. VEGF Trap (low) \\
\hline VEGF Trap (2.5mg/kg) + RT & 12.0 & $(9-14)$ & 6.3 & 0.003 vs. RT, 0.06 vs. VEGF Trap (low) \\
\hline VEGF Trap (25 mg/kg) + RT & 5.0 & $(2-7)$ & 15.5 & 0.001 vs. RT, 0.417 vs. VEGF Trap (high), 0.96 vs. VEGF Trap (low) + RT \\
\hline Abbreviations: \% = daily\% increase in tumor volume; CI = confidence interval; RT = radiation; VEGF = vascular endothelial growth factor; T2x = average doubling time for tumor volume (in days).
\end{tabular}

suggests that low-dose VEGF Trap in combination with single-dose radiation has an enhanced effect on tumor cell kill. It was thought that this enhancement might be improved by varying dose and scheduling protocol. Additional studies were carried out in which low-dose VEGF Trap at $2.5 \mathrm{mg} / \mathrm{kg}$ was compared with an intermediate dose of $10 \mathrm{mg} / \mathrm{kg}$ (because VEGF Trap at $25 \mathrm{mg} / \mathrm{kg}$ appeared to have masked any additional benefit of radiation in enhancing tumor control) in combination with a more clinically relevant fractionated radiotherapy protocol. The results of these studies are reported in the following sections.

\section{Effect of VEGF Trap and fractionated radiation on U87 tumor growth inhibition}

VEGF Trap given before fractionated radiation: in this protocol, VEGF Trap was administered 7 days before radiation. The analyses allowed for separate tumor growth rates in the first and second periods (preradiation: Days -7 to 0 ; postradiation: Days $0+$ ) for the groups that received radiation. The study's main aim was to compare tumor growth rates across treatment groups in the latter period, when all treatments had been applied. Table 2 summarizes the results of the tumor growth modeling analyses during this main study phase, and Fig. 4 presents the original animal data. The low-dose VEGF Trap group $(2.5 \mathrm{mg} / \mathrm{kg}$ every third day, starting at Day -7$)$ demonstrated a reduction in daily percent increase in tumor volume ( $12 \%$ vs. $27 \%$ for control; $p=0.001)$ that was similar to the first single-dose radiation study, whereas the high-dose VEGF Trap group (10 $\mathrm{mg} / \mathrm{kg}$ every third day, starting at Day -7) had an even stronger effect $(7 \%)$ that, again, was similar in trend to the first study. In the radiation only group, tumor daily growth was slowed to $11 \%$ ( $p<0.001$ vs. control). Although low-dose VEGF Trap was comparable to radiation alone ( $p=$ 0.59 ), the combination of low-dose VEGF Trap with radiation (7\% average daily percent increase in tumor volume, Table 1$)$ was significantly better than either radiation alone $(p=0.036)$ or low-dose VEGF Trap alone $(p$ $<0.005)$. The combination of high-dose VEGF Trap with radiation $(5 \%$ average percent daily increase in tumor volume) was also significantly better than radiation alone $(p=0.002)$ but not significantly better than high dose VEGF Trap alone $(p=0.33)$.

VEGF Trap given concurrently with fractionated radiation: Table 3 summarizes the results of the tumor growth modeling analyses based on original animal data shown in Fig. 5. High-dose VEGF Trap was significantly better than radiation in reducing daily percent increase in tumor volume ( $8.5 \%$ vs. $16.1 \%$ for radiation, $p=0.001$ ). The combination of low-dose VEGF Trap with radiation (12\% average daily increase in tumor volume) was significantly better than either radiation alone $(p=0.029)$ or low-dose VEGF Trap alone $(p=0.012)$. The combination of high-dose VEGF Trap (10 mg/kg) with radiation (7\% average daily increase in tumor volume) was also significantly better than radiation alone ( $p=0.001)$ but not high-dose VEGF Trap alone $(p=0.417)$.

VEGF Trap given postradiation: Table 4 summarizes the results of the tumor growth modeling analyses based on original animal data shown in Fig. 6. The results of this schedule followed the same pattern as seen in the previous two schedules with fractionated radiation as well as the first experiment with single-dose radiation. The benefit of combining VEGF Trap with radiation compared with single-modality treatments was once again seen with low-dose VEGF Trap plus radiation. High-dose VEGF Trap at $10 \mathrm{mg} / \mathrm{kg}$ plus radiation significantly reduced percent daily increase in tumor volume when compared with radiation alone but was not significantly different from VEGF Trap alone $(p=0.187)$.

In summary, improved tumor control was seen when radiation (either single dose or fractionated doses) were combined with the lowest dose of VEGF Trap $(2.5 \mathrm{mg} / \mathrm{kg})$ used in these studies. Scheduling did not significantly affectthe efficacy of combined therapy. The relative benefits of combined low-dose VEGF Trap plus fractionated radiation relative to radiation as judged by percent reduction in average daily increase in tumor volume were $36 \%$ for VEGF Trap given before radiation, $27 \%$ for

Table 2. VEGF Trap initiated before fractionated radiation: Summary of tumor growth (Schedule II)

\begin{tabular}{lclll}
\hline Treatment & $\% \Delta$ & $\mathbf{( 9 5 \%} \mathbf{C I})$ & T2x & \multicolumn{1}{c}{$p$ values } \\
\hline Control (human FC protein) & 27.0 & $(23-31)$ & 3.0 & \\
\hline RT (3 x 5 Gy) & 11.0 & $(8-15)$ & 6.5 & 0.001 vs. control, 0.59 vs. VEGF Trap (low), 0.027 vs. VEGF Trap (high) \\
\hline VEGF Trap 2.5 mg/kg) & 12.0 & $(10-15)$ & 5.9 & 0.001 vs. control, 0.59 vs. RT \\
\hline VEGF Trap 10 mg/kg) & 7.0 & $(4-9)$ & 11 & 0.001 vs control, 0.027 vs. RT, 0.001 vs. VEGF Trap (low) \\
\hline VEGF Trap (low) + RT & 7.0 & $(4-9)$ & 10.6 & 0.034 vs. RT, 0.004 vs. VEGF Trap (low) \\
\hline VEGF Trap (high) + RT & 5.0 & $(2-7)$ & 15.3 & 0.002 vs. RT, 0.33 vs. VEGF Trap (high) \\
\hline
\end{tabular}

Abbreviations: $\% \Delta=$ daily $\%$ increase in tumor volume; $\mathrm{CI}=$ confidence interval; $\mathrm{RT}=$ radiation; $\mathrm{VEGF}=$ vascular endothelial growth factor; $\mathrm{T} 2 \mathrm{x}=$ average doubling time for tumor volume (in days). 
Table 3. Trap sequenced concurrently with radiation: Summary of tumor growth (Schedule III)

\begin{tabular}{|c|c|c|c|c|}
\hline Treatment & $\% \Delta$ & $(95 \% \mathrm{CI})$ & $\mathrm{T} 2 \mathrm{x}$ & $p$ values \\
\hline Control (human FC protein) & 27.0 & $(24-30)$ & 2.9 & \\
\hline RT ( 3 x 5 Gy) & 16.0 & $(13-19)$ & 4.6 & 0.001 vs. control, 0.729 vs. VEGF Trap (low), 0.001 vs. VEGF Trap (high) \\
\hline VEGF Trap $(2.5 \mathrm{mg} / \mathrm{kg})$ & 17.0 & $(14-19)$ & 4.5 & 0.001 vs. control, 0.729 vs. RT alone \\
\hline VEGF Trap (10 mg/kg) & 8.5 & $(6-11)$ & 8.5 & 0.001 vs. control, 0.001 vs. RT alone, 0.001 vs. VEGT Trap (low) \\
\hline VEGF Trap (low) + RT & 12.0 & $(9-14)$ & 6.3 & 0.020 vs. RT, 0.008 vs. VEGF Trap (low) \\
\hline VEGF Trap (high) + RT & 7.0 & $(5-9)$ & 10.3 & 0.001 vs. RAD, 0.392 vs. VEGF Trap (high), 0.014 vs. VEGF Trap (low) + RT \\
\hline
\end{tabular}

Abbreviations: $\% \Delta$ daily $\%$ increase in tumor volume; $\mathrm{CI}=$ confidence interval; $\mathrm{RT}=$ radiation; $\mathrm{VEGF}=$ vascular endothelial growth factor; $\mathrm{T} 2 \mathrm{x}=$ average doubling time for tumor volume (in days).

concurrent treatment, and $32 \%$ for drug given postradiation treatment. Although high-dose VEGF Trap (either $10 \mathrm{mg} / \mathrm{kg}$ or $25 \mathrm{mg} / \mathrm{kg}$ ) significantly reduced tumor growth over that of radiation alone, there was no added benefit to combining high dose VEGF Trap with radiation.

\section{Effect of VEGF Trap and radiation on microvessel density}

Immunoassaying for endothelial cells with PECAM-1 revealed an inhibition of tumor angiogenesis 3 weeks after treatment with VEGF Trap or VEGF Trap and radiation. Tumor MVD was similar in the control and radiation-treated tumors. Tumor MVD in the VEGF Trap treated tumors was decreased to between $43 \%$ to $57 \%$ of control or radiationtreated tumors $(p=0.06)$. Tumor MVD in VEGF and radiation-treated groups decreased to between $15 \%$ and $30 \%$ of control or radiationtreated groups ( $p=0.001$ ) (Fig. 7). There was no significant difference in MVD between high-dose VEGF Trap-treated with radiation vs. high dose VEGF Trap alone $(p=0.29)$. However, there was a significant difference in MVD between low-dose VEGF Trap-treated with radiation and low-dose VEGF Trap alone ( $p=0.01$, Fig. 8 ).

\section{8-fluorodeoxyglucose-PET imaging of VEGF Trap-treated tumors}

Figure $9 \mathrm{a}$ illustrates a series of images from a representative, untreated mouse. Figure $9 \mathrm{~b}$ represents a series of images from a representative mouse treated with VEGF Trap dosed at or $10 \mathrm{mg} / \mathrm{kg}$ every 3 days (starting at Day 0) for 3 weeks. Tumor volume $\left(\mathrm{mm}^{3}\right)$ and days following start of treatment are indicated. Because of the difficulty in matching tumor volumes and time after treatment, the percent of metabolically inactive tumor volume (as measured by FDG uptake) was measured as a function of tumor volume and averaged over a range of tumor volumes between 900 and $1,600 \mathrm{~mm}^{3}$. The percent of metabolically inactive tumor was significantly less in untreated tumors $(2.46 \% \pm 0.18 \%)$ than in tumors treated with $10 \mathrm{mg} / \mathrm{kg}$ VEGF Trap $(8.7 \pm 1.26 \%, p=0.01)$ but not significantly different from tumors treated with $2.5 \mathrm{mg} / \mathrm{kg}$ VEGF Trap $(3.36 \pm 0.36 \%, p=0.13)$.

\section{Discussion}

This work demonstrated that VEGF Trap alone is an effective dosedependent inhibitor of tumor growth in U87GBM. These findings agreed with previous studies of VEGF Trap in other preclinical animal models demonstrating efficacy in halting angiogenesis and shrinking tumors $^{30}$. Because VEGF Trap was very potent by itself and could have potentially masked any additional benefits of radiation, both low-dose and high-dose scheduling of the drug were used with radiotherapy. In all scheduling protocols that were investigated, the combination of low-dose VEGF Trap with radiation was significantly better than either treatment modality alone. On the other hand, high-dose VEGF Trap was significantly better than radiation alone and therefore masked any additional benefit that may have resulted from combination therapy.

The benefit of combined treatment with low VEGF Trap and radiation relative to radiation alone was not influenced by scheduling protocol. This result was in contrast to earlier work demonstrating improved radiation response when a VEGFR2 blocker, DC101, was given 4 to 6 days before radiotherapy ${ }^{32}$. This earlier work suggested that tumor vasculature normalization occurred during pretreatment with the VEGFR2 blocker, a process in which pruning of immature and inefficient blood vessels occurs leading to improved tumor perfusion and oxygenation and improved radiation response. The current observations may reflect the absence of a normalization effect by VEGF Trap on U87 GBM vasculature or a missed window of opportunity for normalization because of the particular protocols used in this work. Because it is not known how tumor oxygenation levels may have varied throughout the course of combined treatment with VEGF Trap and radiation, additional studies are warranted to resolve the issue of normalization.

The observation that scheduling did not have an impact on efficacy of combined treatment with VEGF Trap and radiation in this study is also in contrast to recent studies in which VEGF blockade was obtained either by a VEGF receptor2 tyrosine kinase inhibitor, ZD6474, or indirectly by

Table 4. VEGF Trap sequenced post-fractionated radiation: Summary of tumor growth (Schedule IV)

\begin{tabular}{|c|c|c|c|c|}
\hline Treatment & $\% \Delta$ & $(95 \% \mathrm{CI})$ & $\mathrm{T} 2 \mathrm{x}$ & $p$ values \\
\hline Control (human FC protein) & 31.5 & $(28-35)$ & 2.5 & \\
\hline RT (3 x 5 Gy) & 15.0 & $(13-17)$ & 5.1 & 0.001 vs. control, 0.460 vs. VEGF Trap (low), 0.001 vs. VEGF Trap (high) \\
\hline VEGF Trap $(2.5 \mathrm{mg} / \mathrm{kg})$ & 16.0 & $(13-19)$ & 4.7 & 0.001 vs. control, 0.460 vs. RT alone \\
\hline VEGF Trap (10 mg/kg) & 8.0 & $(5-10)$ & 9.2 & 0.001 vs. control, 0.001 vs. RT alone, 0.001 vs. VEGT Trap (low) \\
\hline VEGF Trap (low) + RT & 10.0 & $(7-12)$ & 7.4 & 0.011 vs. RT, 0.001 vs. VEGF Trap (low) \\
\hline VEGF Trap (high) + RT & 5.5 & (3-8) & 12.8 & 0.001 vs. RT, 0.187 vs. VEGF Trap (high), 0.013 vs. VEGF Trap (low) + RT \\
\hline
\end{tabular}

Abbreviations: $\% \Delta$ daily $\%$ increase in tumor volume; $\mathrm{CI}=$ confidence interval; $\mathrm{RT}=$ radiation; VEGF = vascular endothelial growth factor; $\mathrm{T} 2 \mathrm{x}=$ average doubling time for tumor volume (in days). 


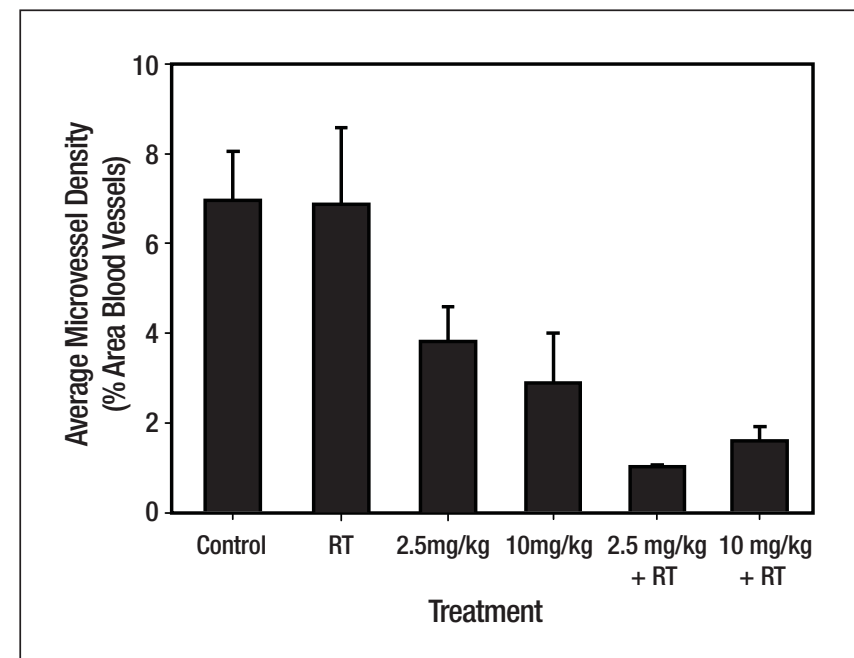

Figure 7. Effect of vascular endothelial growth factor (VEGF) Trap and radiation (RT) (Schedule II) on microvessel density (MVD). Tumor MVD in VEGF Trap-treated tumors was decreased to between $43 \%$ and $57 \%$ of control or RT-treated tumors $(p=0.06)$. Tumor MVD in VEGF Trap and RT-treated groups decreased to between $15 \%$ and $30 \%$ of control or RT-treated groups ( $p=0.001)$. There was no significant difference in MVD between VEGF Traptreated (high dose) + radiation vs. VEGF Trap (high dose) alone $(p=0.29)$. However, there was a significant difference in MVD between VEGF Trap (low dose) + radiation and VEGF Trap (low dose) alone $(p=0.01)$.

HIF-1 alpha blockade of VEGF secretion. In both these studies, optimal antitumor efficacy was obtained when VEGF blockers were sequenced following radiation ${ }^{2,33}$. These studies suggested that prolonged suppression of radiation-induced angiogenesis account for enhanced efficacy of combined treatments with angiogenesis blockade and radiation. However, it is not clearly understood why there is a difference in the impact of scheduling among these agents.

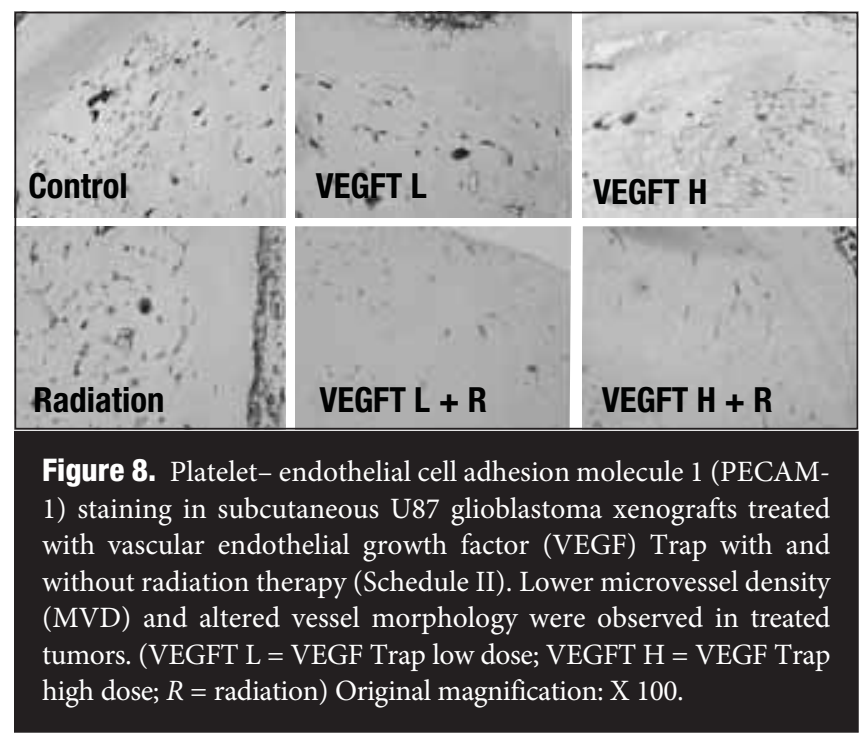

This work is encouraging in that it demonstrates for the first time a benefit in combining VEGF Trap with ionizing radiation in a highly resistant GBM tumor model. VEGF Trap is a unique human fusion protein with very potent binding affinity for VEGF A isoforms as well as placental growth factor (PIGF) and is currently in clinical trials. Its affinity for VEGF is potentially 100- to 1,000-fold higher than existing VEGF monoclonal antibodies such as bevacizumab ${ }^{34}$. This high-affinity blockade of VEGF differentiates VEGF Trap from other anti-VEGF strategies and therefore gives this drug the potential to enhance combination modality treatment with lower dosing.

Mechanisms of enhanced U87 tumor control by combined therapy with VEGF Trap and radiation most likely include inhibition of radiationinduced angiogenesis by VEGF Trap sequestration of circulating VEGF in the bloodstream and in the extracellular tumor space resulting from radiation-induced secretion. Indeed, in this study, a radiation-dosedependent increase in VEGF secretion by U87 glioblastoma cells was observed and excess VEGF was bound in the presence of VEGF Trap. In addition, immunohistochemical findings indicated a reduction in MVD 3 weeks following treatment with VEGF Trap and radiation. Inhibition of radiation-induced angiogenesis was also observed indirectly through FDG-PET imaging, which revealed an increase in metabolically inactive tumor tissue after VEGF Trap treatment, possibly arising from the induction of tumor necrosis or apoptosis in the presence of angiogenesis inhibition. It is also of interest that in this study, a brief period of fraction-

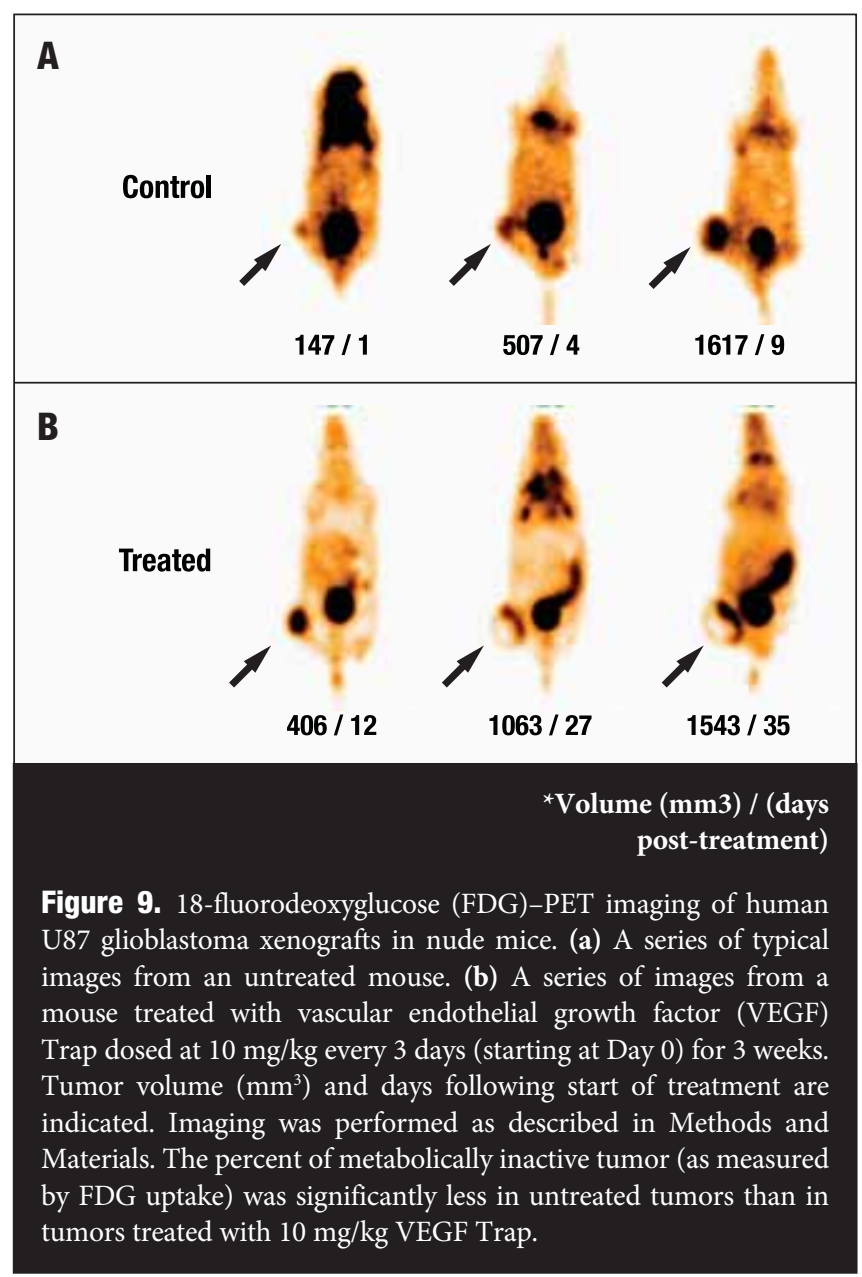


ated radiotherapy with VEGF Trap resulted in tumor growth retardation but not remission. The lack of remission is probably related to continued production of VEGF after removal of drug and radiation and points to the need for chronic therapy with VEGF Trap, which is in agreement with what has been observed for the transient effects of other antiangiogenic agents on tumor control ${ }^{23,35}$.

In conclusion, these studies demonstrate that the combination of lowdose VEGF Trap and radiation is clearly better than radiation alone in a U87 subcutaneous xenograft model. Although high doses of VEGF Trap alone are highly efficacious, it is unclear whether such high doses can be used clinically without incurring normal tissue toxicities. Thus, information on lower doses of VEGF Trap and ionizing radiation are of clinical relevance.

It is understood that the SC xenograft model used in this study has shortcomings in that ectopic tumors implanted SC in the hind limb of animals do not duplicate the vascular microenvironment of orthotopic brain implants $\mathrm{s}^{36}$. However, the use of hind limb injection is the standard approach for xenograft studies with radiation. In addition, human xenografts in immunocompromised nude mice, whether they be ectopic or orthotopic, both have deficiencies in that they can only approximate the human patient situation and seldom reflect accurately the glioblastoma multiforme histopathology seen in patients. This study is encouraging in that it demonstrates for the first time a benefit in combining VEGF Trap with ionizing radiation and warrants further investigations both preclinically and clinically.

\section{References}

1. Garcia-Barros M, Paris F, Cordon-Cardo C, et al. Tumor response to radiotherapy regulated by endothelial cell apoptosis. Science 2003;300:1155-1159.

2. Moeller BJ, Dreher MR, Rabbani ZN, et al. Pleiotropic effects of HIF-1 blockade on tumor radiosensitivity. Cancer Cell 2005;8:99-110.

3. Paris F, Fuks Z, Kang A, et al. Endothelial apoptosis as the primary lesion initiating intestinal radiation damage in mice. Science 2001;293:293-297.

4. Dent $\mathrm{P}$, Yacoub A, Contessa J, et al. Stress and radiation-induced activation of multiple intracellular signaling pathways. Radiat Res 2003;159:283-300.

5. Lanza-Jacoby S, Dicker AP, Miller S, et al. Cyclooxygenase (COX)-2-dependent effects of the inhibitor SC236 when combined with ionizing radiation in mammary tumor cells derived from HER-2/neu mice. Mol Cancer Ther 2004;3:417- 424.

6. Wachsberger P, Burd Marreron R, Rossetti D, et al. Improvement of fractionated radiation therapy by combination with a VEGF blocker, VEGF Trap. 96th Annual Meeting of the American Association of Cancer Research, Anaheim, CA, 2005.

7. Ferrara N. Role of vascular endothelial growth factor in regulation of physiological angiogenesis. Am J Physiol Cell Physiol 2001;280:C1358-C1366.

8. Benjamin LE, Golijanin D, Itin A, et al. Selective ablation of immature blood vessels in established human tumors follows vascular endothelial growth factor withdrawal. J Clin Invest 1999;103:159 -165 .

9. Tran J, Master Z, Yu JL, et al.A role for survivin in chemoresistance of endothelial cells mediated by VEGF. Proc Natl Acad Sci U S A 2002;99:4349-4354.

10. Moeller BJ, Cao Y, Li CY, et al. Radiation activates HIF-1 to regulate vascular radiosensitivity in tumors: role of reoxygenation, free radicals, and stress granules. Cancer Cell 2004;5:429-441.

11. Hicklin DJ, Ellis LM. Role of the vascular endothelial growth factor pathway in tumor growth and angiogenesis. J Clin Oncol 2005;23:1011-1027.

12. Asano M, Yukita A, Matsumoto T, et al. An anti-human VEGF monoclonal antibody, MV833, that exhibits potent anti-tumor activity in vivo. Hybridoma 1998;17:185-190.

13. Kamiya K, Konno H, Tanaka T, et al. Antitumor effect on human gastric cancer and induction of apoptosis by vascular endothelial growth factor neutralizing antibody. Jpn J Cancer Res 1999;90:794-800

14. Kim ES, Serur A, Huang J, et al. Potent VEGF blockade causes regression of coopted vessels in a model of neuroblastoma. Proc Natl Acad Sci U.S.A. 2002;99:11399 -11404.

15. Willett CG, Boucher Y, di Tomaso E, et al. Direct evidence that the VEGF-specific antibody bevacizumab has antivascular effects in human rectal cancer. Nat Med 2004;10:145-147.
16. Abdollahi A, Lipson KE, Han X, et al. SU5416 and SU6668 attenuate the angiogenic effects of radiation-induced tumor cell growth factor production and amplify the direct antiendothelial action of radiation in vitro. Cancer Res 2003;63:3755-3763.

17. 17. Drevs J, Hofmann I, Hugenschmidt H, et al. Effects of PTK787/ZK 222584, a specific inhibito of vascular endothelial growth factor receptor tyrosine kinases, on primary tumor, metastasis, vessel density, and blood flow in a murine renal cell carcinoma model. Cancer Res 2000;60:48194824.

18. Fong TA, Shawver LK, Sun L, et al. SU5416 is a potent and selective inhibitor of the vascular endothelial growth factor receptor (Flk-1/KDR) that inhibits tyrosine kinase catalysis, tumor vascularization, and growth of multiple tumor types. Cancer Res 1999;59:99-106.

19. Wedge SR, Ogilvie DJ, Dukes M, et al. ZD4190: An orally active inhibitor of vascular endothelial growth factor signaling with broad-spectrum antitumor efficacy. Cancer Res 2000;60:970-975

20. Hasumi Y, Mizukami H, Urabe M, et al. Soluble FLT-1 expression suppresses carcinomatous ascites in nude mice bearing ovarian cancer. Cancer Res 2002:62:2019 -2023.

21. Holash J, Davis S, Papadopoulos N, et al. VEGF-Trap: a VEGF blocker with potent antitumor effects. Proc Natl Acad Sci U S A 2002;99:11393-11398.

22. Kerbel R, Folkman J. Clinical translation of angiogenesis inhibitors. Nat Rev Cancer 2002;2:727739.

23. Wachsberger P, Burd R, Dicker AP. Tumor response to ionizing radiation combined with antiangiogenesis or vascular targeting agents: exploring mechanisms of interaction. Clin Cancer Res 2003;9:1957-1971.

24. Wachsberger P, Burd R, Dicker AP. Improving tumor response to radiotherapy by targeting angiogenesis signaling pathways. Hematol Oncol Clin North Am 2004;18:1039-1057, viii.

25. Vajkoczy P, Schilling L, Ullrich A, et al. Characterization of angiogenesis and microcirculation of high-grade glioma: an intravital multifluorescence microscopic approach in the athymic nude mouse. J Cereb Blood Flow Metab 1998;18:510-520.

26. Ferrara N, Chen H, Davis-Smyth T, et al. Vascular endothelial growth factor is essential for corpu luteum angiogenesis. Nat Med 1998;4:336 -340.

27. Gerber HP, Kowalski J, Sherman D, et al. Complete inhibition of rhabdomyosarcoma xenograft growth and neovascularization requires blockade of both tumor and host vascular endothelial growth factor. Cancer Res 2000:60:6253- 6258.

28. Gerber HP, Hillan KJ, Ryan AM, et al. VEGF is required for growth and survival in neonatal mice. Development 1999;126:1149-1159.

29. Kuo CJ, Farnebo F, Yu EY, et al. Comparative evaluation of the antitumor activity of antiangiogenic proteins delivered by gene transfer. Proc Natl Acad Sci U S A 2001;98:4605- 4610.

30. Konner J, Dupont J. Use of soluble recombinant decoy receptor vascular endothelial growth factor trap (VEGF Trap) to inhibit vascular endothelial growth factor activity. Clin Colorectal Cancer 2004;4(Suppl. 2):S81-S85.

31. 31. Huang J, Frischer JS, Serur A, et al. Regression of established tumors and metastases by poten vascular endothelial growth factor blockade. Proc Natl Acad Sci U S A 2003;100:7785-7790.

32. Winkler F, Kozin SV, Tong RT, et al. Kinetics of vascular normalization by VEGFR2 blockad governs brain tumor response to radiation: role of oxygenation, angiopoietin-1, and matrix metalloproteinases. Cancer Cell 2004;6:553-563.

33. Williams KJ, Telfer BA, Brave S, et al. ZD6474, a potent inhibitor of vascular endothelial growth factor signaling, combined with radiotherapy: schedule-dependent enhancement of antitumor activity. Clin Cancer Res 2004;10:8587- 8593.

34. Bergsland EK. Update on clinical trials targeting vascular endothelial growth factor in cancer. Am J Health Syst Pharm 2004;61:S12-S20.

35. Klement G, Baruchel S, Rak J, et al. Continuous low-dose therapy with vinblastine and VEGF receptor-2 antibody induces sustained tumor regression without overt toxicity. J Clin Invest 2000;105:R15-R24.

36. Blouw B, Song $\mathrm{H}$, Tihan $\mathrm{T}$, et al. The hypoxic response of tumors is dependent on their microenvironment. Cancer Cell 2003;4:133-146. 